\title{
CDK1/2/4 Inhibitor AG-024322
}

National Cancer Institute

\section{Source}

National Cancer Institute. CDK1/2/4 Inhibitor AG-024322. NCI Thesaurus. Code C52182.

A cyclin-dependent kinase (CDK) inhibitor with antineoplastic activity. AG-024322 selectively inhibits cyclin-dependent kinases (particularly CDK1,2 and 4), enzymes that regulate cell cycle prog ression. Inhibition of CDK may result in cell cycle arrest, induction of apoptosis, and inhibition of DNA replication and tumor cell proliferation. 